\title{
Optical alignment of a cylindrical object
}

\author{
Chaolong Song, Nam-Trung Nguyen*, Anand Krishna Asundi \\ School of Mechanical and Aerospace Engineering, Nanyang Technological University, \\ 50 Nanyang Avenue, Singapore 639798, Singapore \\ *E-mail: mntnguyen@ntu.edu.sg; Tel: (+65) 6790 4457; Fax: (+65) 67924062
}

\begin{abstract}
This paper reports the use of theory of geometrical optics to analyze how an optical field interacts with a cylindrical object. Of great interest is the mechanism how a laser beam with a special profile manipulates a particle which has a similar shape as the beam profile. The present paper investigates the interaction between a cylinder-shape fiber and a laser beam with a line-shape profile. Based on the Fresnel equation, a numerical model was formulated to describe the optical torque generated by a projected line-shape optical image. The drag force was also considered in the model to accurately describe the fiber's movement in a liquid. A differential equation is established to describe this damped movement of the cylinder. Parametric analysis was carried out to investigate the influence of the beam power and the liquid viscosity as well as the density, the length and the diameter of the cylindrical object. The movement of a carbon fiber was measured with a CCD camera. The observed experimental results agree well with the theoretical results.
\end{abstract}

Keywords: Optical tweezers, line-shape profile, cylinder, trapping dynamics, optical alignment, geometrical optics 


\section{Introduction}

The manipulation and trapping of microparticles by a laser beam was first reported by Ashkin [1, 2] in the early 1970s. He showed the interaction between the propagating photons and the microparticles, and found that the radiation pressure can overcome gravity and levitate the micorparticles. Ashkin [3] extended his work to threedimensional trapping of microparticles by a tightly focused laser beam, now well known as optical tweezers. Optical tweezers have attracted worldwide attention for the last 20 years because they provide a non-invasive and precise way to manipulate objects at the microscale. This technique has been greatly enhanced by the development of multiple beam trapping [4], the scanning beam trapping system [5] and holography tweezers [6]. These improvements allow optical tweezers to manipulate multiple particles simultaneously.

Another interesting application is how to achieve more complicated movements of a single particle, such as alignment or rotation, using optical tweezers. One simple technique is to utilize a circularly polarized laser beam to impart angular momentum to birefringent particles, which would rotate as a result [7]. Different beam profiles have also been employed in such a particle rotation system. Laguerre-Gaussian modes generated by holography were demonstrated to trap $\mathrm{CuO}$ particles [8]. Later works showed that this orbital angular momentum could add to or subtract from the spin component associated with polarization [9]. Further more, Bessel beams showed the possibility of aligning micro-sized rod along the axis of the beam [10]. To the best knowledge of the authors, most studies of optical alignment or rotation have been based on the transfer of angular momentum. Although the optical torque has been calculated mathematically by Nieminen et al [11], as yet few experiments showed the quantitative relationship between the angular velocity of the particle and the optically driving torque. One of the reasons might be the asymmetric shape of the particles used in the experiments. Methods of optical alignment or rotation by using non-circular shape beam profiles have also been reported. Ting et al added a cylindrical lens to a typical optical tweezers system to change the intensity distribution of the laser beam [12]. Marenori et al 
showed that an elliptically shaped laser beam spot can also trap and align slender particles by using a liquid crystal device [13]. Although these studies did not go further into the mathematical description of alignment or rotation movement, they reveal a growing motivation to investigate the mechanism with which a laser beam with special beam profile manipulates the particles which have a similar shape as the beam.

This paper presents a model and the parametric study on the optical alignment of a cylindrical object by a laser beam with a line-shape profile. We assumed for our model the Mie regime, in which the focused laser beam is described as a bundle of individual optical rays, whose propagation directions are determined by the numerical aperture of objective lens. These rays will be reflected and refracted by an object. Both reflection and refraction mean changes in propagation direction of the rays and thus in their momentum. Momentum conservation law ensures that the object must receive a transferred momentum from the rays. To understand the total effect from each individual ray given to the cylindrical object in our case, a single-ray analysis was carried out. Assuming that the interaction between the cylindrical object and the light is in the Mie regime, a numerical simulation was carried out to describe the optical torque generated by a projected line-shape image based on the Fresnel equation. A force balance was used for modeling the fiber's movement in water, where the drag force is considered as the damping force. A second-order ordinary differential equation is established based on this force balance. An experiment was carried out to verify the proposed theory.

\section{Theoretical analysis}

When the size of an object is much larger than the wavelength of the laser light, the interaction between the object and the incident light can be described by using geometrical optics (e.g. a $15 \mu m$ diameter bead trapped in a $\lambda=1064 n m$ laser beam) [14]. In our case, the investigated diameters of the cylindrical objects range from 5 to $15 \mu \mathrm{m}$, and the length is more than $40 \mu m$. The corresponding effective diameter is about $16 \mu m$. In a later experiment, the laser light has a wavelength of $808 \mathrm{~nm}$. Therefore it is 
sufficiently precise to employ ray theory to analyze the energy and momentum transfer between the cylindrical object and the light beam.

We first consider the force exerted on a disk with infinitely small thickness by a single ray of power $P$, figure 1 . This ray is repeatedly reflected and refracted by the disk until the power decreases to zero. Each reflection and refraction will impart a momentum back to the disk as a result of deflection of light propagation. The reflected ray of power $P R$ and all the infinite number of subsequent refracted rays of decreasing power $P T^{2}$, $P T^{2} R, \ldots P T^{2} R^{n}, \ldots$ will give their contributions to the total force $[15,16]$, where $T$ and $R$ are the transmission and reflection coefficients, respectively. This force can be broken into two components: one is along the propagation direction of the incident beam, called the scattering force $F_{Z}$; the other is perpendicular to this direction, named gradient force $F_{y}[17]$.

$$
\begin{aligned}
& F_{z}=F_{s}=\frac{n_{1} p}{c} \times\left\{1+R \cos 2 \theta-\frac{T^{2}[\cos (2 \theta-2 \gamma)+R \cos 2 \theta]}{1+R^{2}+2 R \cos 2 \gamma}\right\} \\
& F_{y}=F_{\mathrm{g}}=\frac{n_{1} p}{c} \times\left\{1+R \sin 2 \theta-\frac{T^{2}[\sin (2 \theta-2 \gamma)+R \sin 2 \theta]}{1+R^{2}+2 R \cos 2 \gamma}\right\}
\end{aligned}
$$

where $\theta$ and $\gamma$ are the angles of incidence and refraction, respectively, which obey Fresnel's law:

$$
\sin \theta n_{1}=\sin \gamma n_{2}
$$

where $n_{1}$ and $n_{2}$ are the refractive indices of the surrounding medium and of the disk respectively. The two components of the optically induced force are summed up over all scattered rays. The light beam is assumed to be circularly polarized and the energy is evenly distributed to two vibration directions. The transmission and reflection coefficients of these two directions, which are parallel as well as perpendicular to the incident plane, are different.

$$
\begin{gathered}
R_{s}=r_{s}^{2} \quad R_{p}=r_{p}^{2} \\
T_{s}=\frac{n_{2} \cos \gamma}{n_{1} \cos \theta} t_{s}^{2} \quad T_{p}=\frac{n_{2} \cos \gamma}{n_{1} \cos \theta} t_{p}^{2}
\end{gathered}
$$


where the subscript $s$ and $p$ denote the perpendicular wave and the parallel wave, respectively. The parameters $r$ and $t$ are the amplitude ratios between the scattered and incident light. These ratios are given by the Fresnel equation:

$$
\begin{array}{cc}
r_{s}=-\frac{\sin (\theta-\gamma)}{\sin (\theta+\gamma)} & r_{p}=\frac{\tan (\theta-\gamma)}{\tan (\theta+\gamma)} \\
t_{s}=\frac{2 \sin \gamma \cos \theta}{\sin (\theta+\gamma)} & t_{p}=\frac{2 \sin \gamma \cos \theta}{\sin (\theta+\gamma) \cos (\theta-\gamma)}
\end{array}
$$

Before considering how the line-shape light image interacts with the cylinder, it is easier first to investigate the force on the object given by a single point spot. Fig. 2(a) and (b) illustrate the side view and top view of the modeled cylinder. The laser beam is assumed to be well focused to form an image point 0 , which is at a distance $s$ to the axis of the cylinder. Assuming a sufficiently long cylinder, the impact by rays in the planes $\beta_{1}$ and $\beta_{2}$, which are symmetrical with reference to plane $\alpha$, would offset each other. Therefore, only the rays in the $\alpha$ plane, which is perpendicular to the axis of the cylinder, contribute to the net force. Basically, the force can be calculated by numerically integrating each impact from a single ray which is scattered by the cylinder.

Next, we consider a line-shape image shown as the bold line in figure 2(c), which shares the same center with the cylinder, but is oriented with an angle $\psi$ to the axis of the cylinder. Since the line-shape image can be considered as an infinitely large number of image points, each image point would exert a net force whose direction is perpendicular to the axis of the cylinder. Therefore each force would result in a torque that rotates the cylinder to align with the line image. The total torque can be retrieved by summing up all the contributions from each image point:

$$
M_{o p t}=\sum_{i=1}^{\infty} \vec{r}_{i} \times \vec{F}_{i}
$$

where $\vec{F}_{i}$ represents a force contributed by a single image point, and $\vec{r}_{i}$ denotes the corresponding moment arm of force. 
Figure 3 shows the optical torque versus the angle between the axis of cylinder and the line-shape image. In this simulation, the cylinder has a diameter of $D=10 \mu m$, a length of $L=40 \mu \mathrm{m}$, and a relative refractive index of $n=1.2$; the length of line-shape image is $l=20 \mu \mathrm{m}$. In figure 3 the value of the optical torque is normalized by its maximum. For different numerical apertures, the maximum value of the torque is achieved with different angles.

If the cylindrical object is immersed in a liquid, such as water, the viscosity of the liquid causes the Stokes drag force, which cannot be neglected. In the later experiment, we found that the cylinder-shape fiber would not rotate unless the initial angle between the axis of the cylinder and the image was about $40^{\circ}$. The cylinder needs a sufficiently large torque to overcome friction and to trigger its movement. Loth calculated the drag force of non-spherical solid particles of regular shape. For a cylinder shape, the drag force can approximately assume the needle model [18]:

$$
F_{D}=3 \pi d_{e} \mu_{f} f_{E} \cdot v
$$

where $d_{e}$ is the effective diameter, $\mu_{f}$ is the viscosity of the liquid, $f_{E}$ is the Stokes correction factor for spheroid, and $v$ is the relative velocity of the cylinder. The effective diameter and the Stokes correction factor are determined as follows:

$$
\begin{gathered}
d_{e}=D \cdot E^{1 / 3} \\
f_{E}=\frac{(4 / 3) E^{2 / 3}}{\ln (2 E)-1 / 2}
\end{gathered}
$$

where $E=\frac{L}{D}$ is the aspect ratio: $L$ is the length of the cylinder, and $D$ is the diameter.

In the model shown in figure 2(c), the driving force is position dependent, and the damping force (drag force) is velocity dependent. Therefore the movement of the cylinder can be formulated by a second-order ordinary differential equation (ODE):

$$
M_{o p t}(\psi)-\zeta \cdot \frac{d \psi}{d t}=I \cdot \frac{d^{2} \psi}{d t^{2}}
$$


The first term at the left-hand side is a driving torque induced optically, $\zeta$ is the damping coefficient induced by the drag force, and $I=m L^{2} / 12$ is the moment of inertia, which is a property of the cylinder itself. The mass of the cylinder is denoted with $m$.

The ODE (10) can be solved numerically. To reflect the real condition of the later experiment, the simulation conditions are chosen as follows: the power of the laser beam $P=20 \mathrm{~mW}$; the numerical aperture of the objective lens $N A=0.85$; the length of lineshape image $l=20 \mu \mathrm{m}$; the viscosity of liquid $\mu=0.894 \times 10^{-3} \mathrm{~Pa} \cdot \mathrm{s}$; the length of the cylindrical object $L=40 \mu \mathrm{m}$, the diameter $D=10 \mu \mathrm{m}$, the density $\rho=1.8 \mathrm{~g} \mathrm{~cm}^{-3}$; and the relative refractive index $n=1$.2. In order to investigate how these parameters impact the movement of cylindrical objects, a parametric study was carried out to look at the response of angular position and angular velocity.

Figures 4 (a) and (b) show the angular positions and angular velocities under different laser beam power. The power of the laser beam has a linear relationship with the optically induced torque, which is the first term in equation (10). At the beginning of movement, the drag force is small due to the low angular velocity. Therefore, the optical torque becomes a dominant factor in this period. A higher laser power would accelerate the cylinder more strongly. When the velocity increases to a higher level, the impact of the drag force becomes significant. A movement with higher velocity would suffer more from friction, and ultimately approaches the end position more quickly.

In equation (10), the optical torque acts as the driving factor, whereas the drag force plays an opposing role. Since the drag force is proportional to the viscosity of the liquid, increasing the viscosity will cause an opposite effect to increasing the laser power. A larger viscosity results in a lower angular velocity during the process of acceleration (figure 5). Results in both figures 4 and 5 share one common feature, namely that a cylinder with a high angular velocity approaches its final position more quickly. This can be explained since at the end of the movement, the influence of the optical torque is insignificant as shown in figure 3. In contrast, the drag force becomes dominant because of the increase in velocity. Thus, the cylinder with a higher angular velocity in the initial 
period would be exposed to a larger drag force, which would make the cylinder appear to be "suddenly stopped".

The inertia of the object is another important factor in a kinetic system; it characterizes the resistance of an object to change its state of motion. The variation of density would directly change the moment of inertia of the object without any influence on the dimensions of the cylinder. The solid line in figure 6 represents the movement of a cylinder with the smallest inertia, which has the largest velocity at the beginning of the rotation. Despite the high velocity, its behavior at the end of movement is different from those caused by variation of the laser power and the viscosity. Although an object with larger inertia has lower velocity at beginning, it approaches its end position more quickly. When the angular velocity has already been accelerated to a high level, the larger inertia makes it much more difficult to slow down. If the inertia is large enough, vibration around the end position is possible, as shown by the dashed line.

The variation of the length of the cylinder would have both influences on both the inertia and the drag force. Although the increase of the length would cause difficulty in changing the cylinder's state of motion because of the larger inertia, it would also result in a larger drag force. Therefore a longer cylinder has a lower angular velocity during the process of acceleration, but it does not quickly approach the final position as in the case of a larger inertia (figure 7). The reason is the larger surface area of the longer cylinder, which results in a stronger drag force to resist the larger inertia.

The diameter of the cylinder is a parameter, which has a relationship with all three terms in equation (10). First, the interaction area between the light beam and the cylinder is affected by the diameter. Then the contact area between the cylinder and the liquid is proportional to this parameter. Last but not least, the moment of inertia also has a linear relationship with the diameter. The solid line in figure 8 represents the movement of a cylinder with $10 \mu m$ diameter, whereas the dot-dashed line represents that with $5 \mu m$. At the initial period of the movement, the cylinder with a larger diameter has a higher angular velocity because more optically induced torque is exerted on the cylinder with a 
larger diameter. But for the cylinder with a larger diameter, the acceleration is limited as a result of the larger inertia and the larger drag force. Therefore, the velocity of the cylinder with $5 \mu m$ diameter can be pushed to a higher level than that of the cylinder with $10 \mu m$ diameter; see figure 8 (b). However, if the diameter of the cylinder is sufficiently large, as represented by the dashed line in figure 8 , both the acceleration and velocity would be reduced to a low level.

\section{Experiment and discussion:}

Figure 9 is a schematic diagram of the optical alignment system used in our experiment. The wavelength of the infrared laser is $808 \mathrm{~nm}$, and the power is $20 \mathrm{~mW}$. The laser beam has a rectangular profile with high aspect ratio. Therefore when the beam is highly focused, a line segment, which has a length $l=20 \mu \mathrm{m}$, is formed at the image plane, as illustrated by cross section A-A in figure 9. A carbon fiber (Toho Tenax America Inc., Rockwood, TN, USA) was used here to mimic the cylinder model. The carbon fibers have different lengths randomly distributed from 10 to $450 \mu \mathrm{m}$. The fiber chosen for our experiments has a length of $L=40 \mu \mathrm{m}$, a diameter $D=10 \mu \mathrm{m}$, and a density of $\rho=$ $1.8 \mathrm{~g} \mathrm{~cm}^{-3}$. The relative refractive index of carbon is approximately $n=1.2$ [19]. The numerical aperture of the inverted objective lens is 0.85 . The laser emits coherent infrared light, which is collimated and expanded by a telescope system. The laser beam arrives at an inverted objective lens after reflection by a beam splitter. The expansion of the beam is intended to fully fill the aperture of the objective lens, which will tightly focus the laser beam to form a line-shape image. This line-shape image interacts with the carbon fiber dispersed in water and sandwiched between two glass slides. The images of the fiber were recorded with a CCD camera (PULNIX TM-765E). The position of the fiber as function of time was subsequently evaluated from the recorded image frames and the known frame rate.

Figure 10 shows the rotation of the fiber under the optically induced torque. The fiber was located in a manner such that the centers of fiber and the line-shape image coincide, and the initial angle between the axis of the fiber and the line is $\psi=47^{\circ}$. Under the driving optical torque, the fiber gradually approaches its final position. 
Figure 11 shows the evaluated position of the fiber as a function of time. As illustrated in figure 11, the slope of the angular position is almost constant, which means that angular velocity of the fiber remained the same during its movement. As the result of the damping liquid, the velocity of rotation becomes more stable. Moreover, as the optically induced torque becomes weaker when the fiber approaches the final position, the drag force would have a dominant impact on the movement. The fiber is stopped without any observed vibration. This vibration-free movement reveals a possibility of actuating the fiber as a micromotor. At the moment the fiber stops, the laser beam with a line-shape profile can be switched off and turned on again with another angle $\psi$. The cylindrical fiber would continue to rotate with the same and almost constant angular velocity. The rotation of the line-shape image can be achieved with a spatial light modulator.

\section{Conclusion}

In conclusion, we investigated the mechanism with which a laser beam with line-shape profile interacts with a micro-sized cylindrical object in the Mie regime. A numerical model was formulated to describe the optical torque generated by the projected line-shape image. A second-order ordinary differential equation was established to describe this damped movement of the cylinder. A parametric analysis was carried out to investigate the influence of the laser beam power, the liquid viscosity, the object density, and object's geometry. The model was verified with experiments using an infrared laser and carbon fibers. The paper presents the feasibility of alignment of long objects using optical tweezers. The results also promise the realization of a micromotor, which may have an application as a stirrer to improve mixing in a microfluidic system. 


\section{References}

[1] Ashkin J M, 1970 Acceleration and trapping of particles by radiation pressure, Phys. Rev. Lett. 24 156-9

[2] Ashkin J M and Dziedzic J 1971 Optical levitation by radiation pressure, Applied Phys. Lett. 19 283-5

[3] Ashkin J M, Dziedzic J M, Bjorkholm J E, and Chu S 1986 Observation of a single-beam gradient force optical trap for dielectric particles, Opt. Lett. 11288 90

[4] Fournier J M, Boer G, Delacrétaz G, Jacquot P, Rohner J and Salathé R P 2004 Building optical matter with binding and trapping forces, Proc. SPIE 5514 309-17

[5] Sasaki K, Koshioka K, and Masuhara H 1991 Laser-scanning micromanipulation and spatial patterning of fine particles, Japan. J. Appl. Phys. 30, L907-9

[6] Dufresne E R, Spalding G C, Dearing M T, Sheets S A, and Grier D G 2001 Computer-generated holographic optical tweezer arrays Rev. Sci. Instrum. 72 $1810-6$

[7] Friese M, Nieminen T A, Heckenberg N R, and Rubinsztein-Dunlop H 1998 Optical alignment and spinning of laser-trapped microscopic particles Nature 394, $348-50$

[8] He H, Friese M E J, Heckenberg NR, and Rubinsztein-Dunlop H 1995 Direct observation of transfer of angular momentum to absorptive particles from a laser beam with a phase singularity, Phys. Rev. Lett. 75 826-9

[9] Simpson N B, Dholakia K, Allen L, and Padgett M J 1997 Mechanical equivalence of spin and orbital angular momentum of light: an optical spanner, Opt. Lett. 22 52-4

[10] Garcés-Chávez V, McGloin D, Melville H, Sibbett W, Dholakia K 2002 Simultaneous micromanipulation in multiple planes using a self-reconstructing light beam, Nature 419 145-7

[11] Nieminen, T A, Parkin S, Heckenberg N R, and Rubinsztein-Dunlop H 2004 Optical torque and symmetry, Proc. SPIE 5514 254-63

[12] Yu T, Cheong F C, and Sow C H 2004 The manipulation and assembly of CuO nanorods with line optical tweezers, Nanotechnology 15 1732-6 
[13] Marenori K., Mao Y., and Susumu S. 2008 Optical particle manipulation using an LC device with eight-divided circularly hole-patterned electrodes, Opt. Express, 16 10059-65

[14] Gregor K, Nieminen T A, Parkin S, Heckenberg N R, and Rubinsztein-Dunplop H 2006 Calculation of optical trapping landscapes, Proc. SPIE 6326 63260K

[15] Roosen G and Imbert C 1976 Optical levitation by means of 2 horizontal laser beams-theoretical and experimental study, Phys. Lett. A 59 6-8

[16] Roosen G 1979 Optical levitation of spheres, Can. J. Phys. 57 1260-79

[17] Ashkin A 1992 Forces of a single-beam gradient laser trap on a dielectric sphere in the ray optics regime, Biophys. J. 61 569-82

[18] Loth E 2007 Drag of non-spherical solid particles of regular and irregular shape, Powder Technology 182 342-53

[19] Pluchino AB, Goldberg SS, Dowling JM, and Randall CM 1980 Refractive-index measurements of single micron-sized carbon particles, Appl. Opt. 19 3370-2 


\section{List of Figures}

Figure $1 \quad$ An incident light beam of power $P$ hits a disk with infinitely small thickness. Because of the momentum transfer, the reflected and refracted light exerts a net force on this disk, which can be broken into two components along the $y$ and $z$ directions.

Figure 2 Model of optical alignment of a cylindrical object. (a) and (b): geometry of a cylinder under the gradient field formed by a single focused laser spot 0 ; (c): The cylinder is placed with an initial angle $\psi$ with reference to the focused line-shape image. The well-focused laser beam creates an optical torque to rotate the cylinder.

Figure 3 The normalized optical torque as a function of angular displacement (the centers of cylinder and the line-shape image coincide, the cylinder has a diameter of $D=10 \mu m$, a length of $L=40 \mu m$, and a relative refractive index of $n=1.2$; the length of the line-shape image is $l=20 \mu m$ ).

Figure 4 Angular positions $\psi$ (a) and angular velocities (b) for different laser beam power.

Figure 5 Angular positions $\psi$ (a) and angular velocities (b) for different viscosities of the surrounding liquid.

Figure 6 Angular positions $\psi$ (a) and angular velocities (b) for different mass densities of the cylinder.

Figure 7 Angular positions $\psi$ (a) and angular velocities (b) for different lengths of the cylinder.

Figure 8 Angular positions $\psi$ (a) and angular velocities (b) for different radii of the cylinder.

Figure $9 \quad$ Schematic setup for optical tweezers and optical alignment. 
Figure 10 The fiber is located in a manner that the centers of fiber and line-shape image coincide, and the initial angle between the axis of fiber and the line is $47^{\circ}$. Under the driving optical torque, the fiber gradually approaches its final position. The time step between the frames is $1.3 \mathrm{~s}$.

Figure 11 Angular position (a) and velocity (b) from the experiment result and numerical simulation. Setting the line-shape image as the reference, a cylinder-shape fiber is initially located with an angle $\psi=47^{\circ}$. Under the driving optically induced torque and the damping torque of the drag force, the fiber rotates until its axis aligns with the line-shape image. After the movement was triggered, rotation took place with an almost angular velocity (illustrated both by experimental and numerical results). Because the damping factor is relatively large in this system, the fiber's movement approaches its final position without much vibration. 


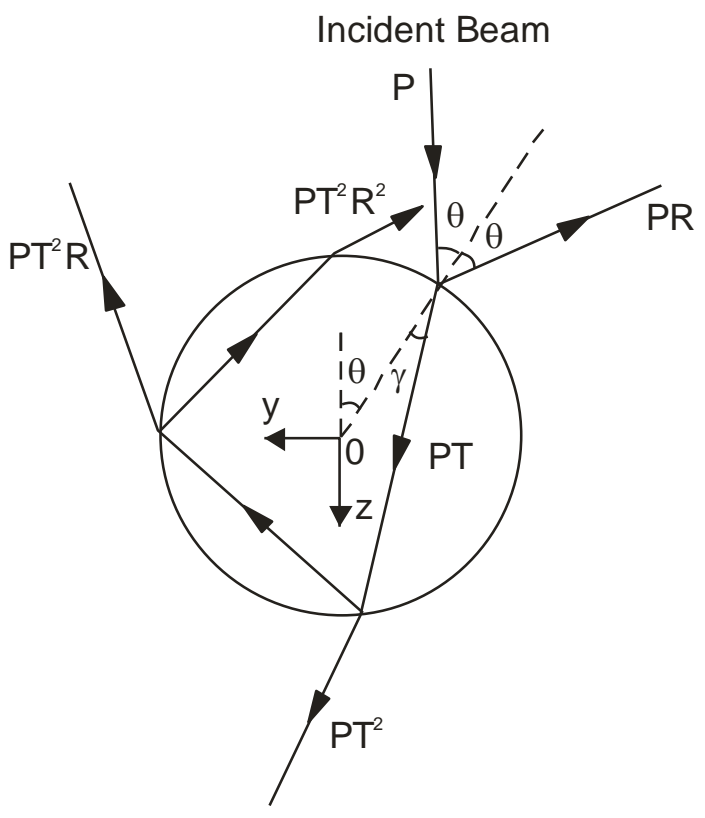

Figure 1. 


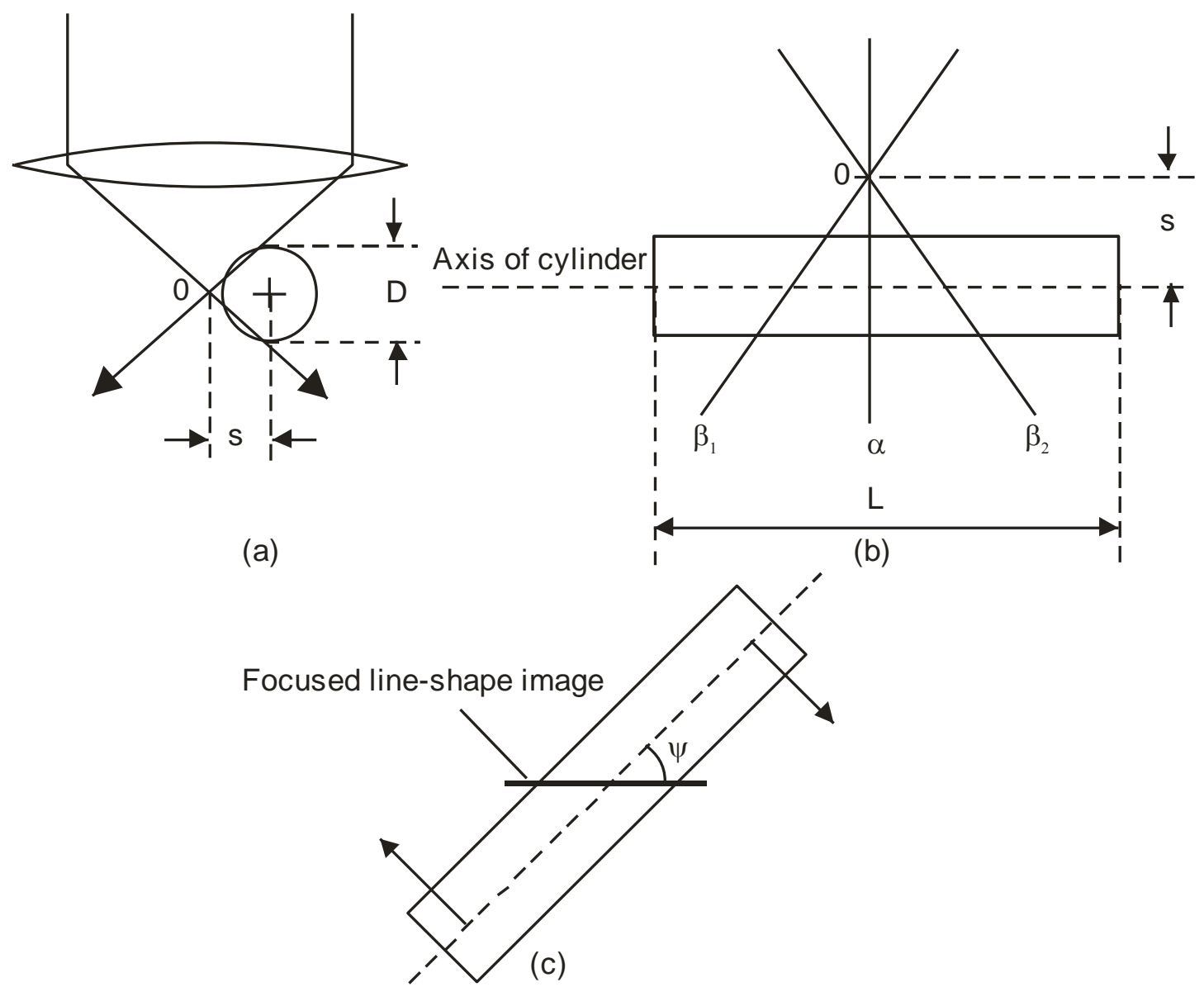

Figure 2. 


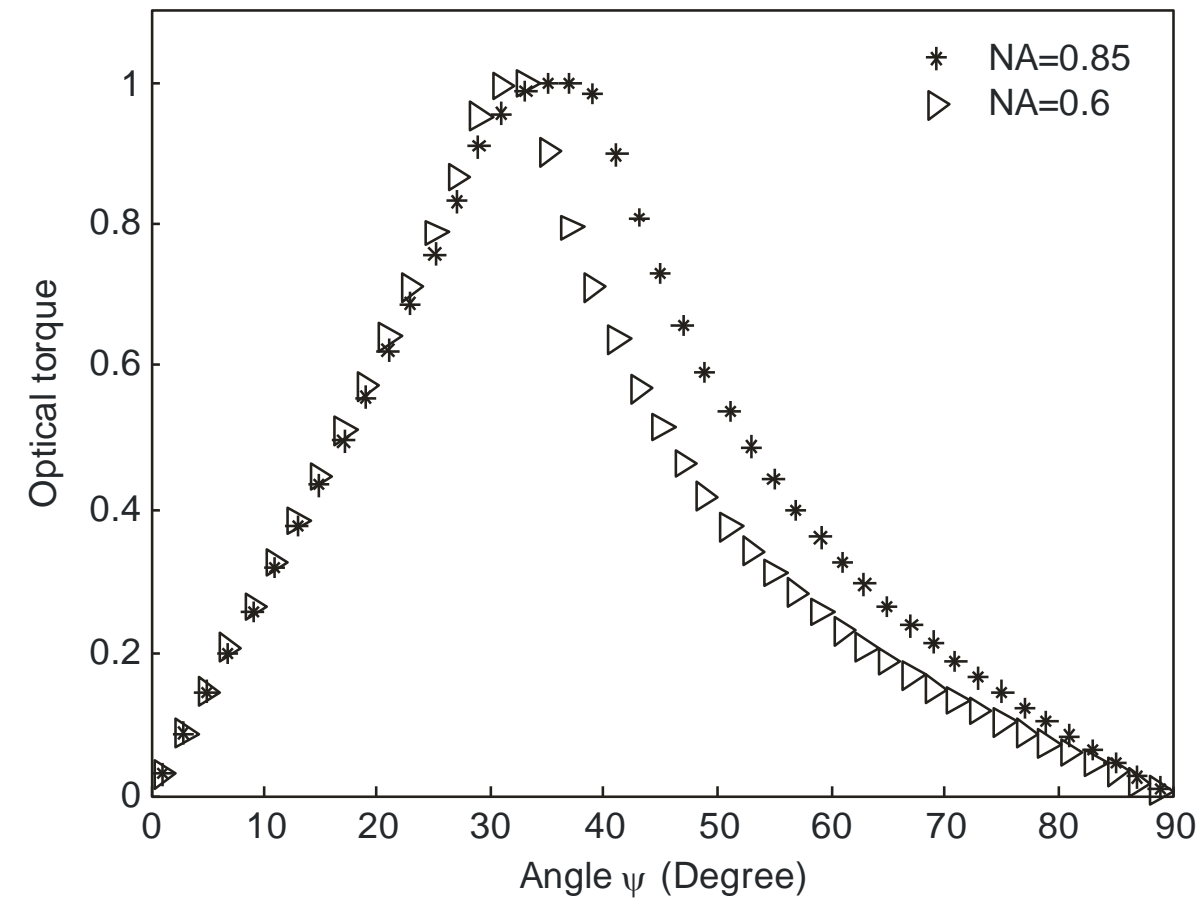

Figure 3. 

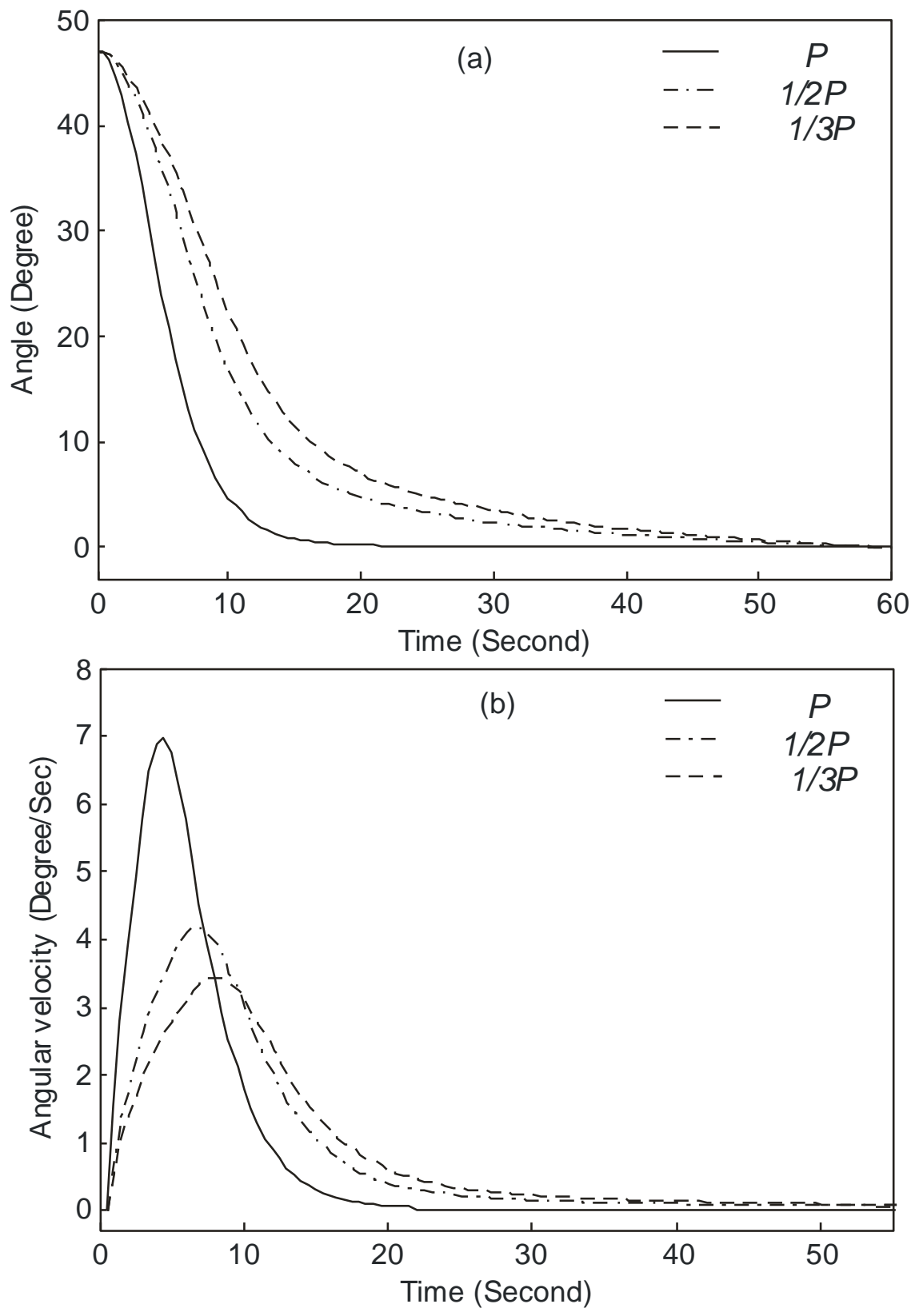

Figure 4. 

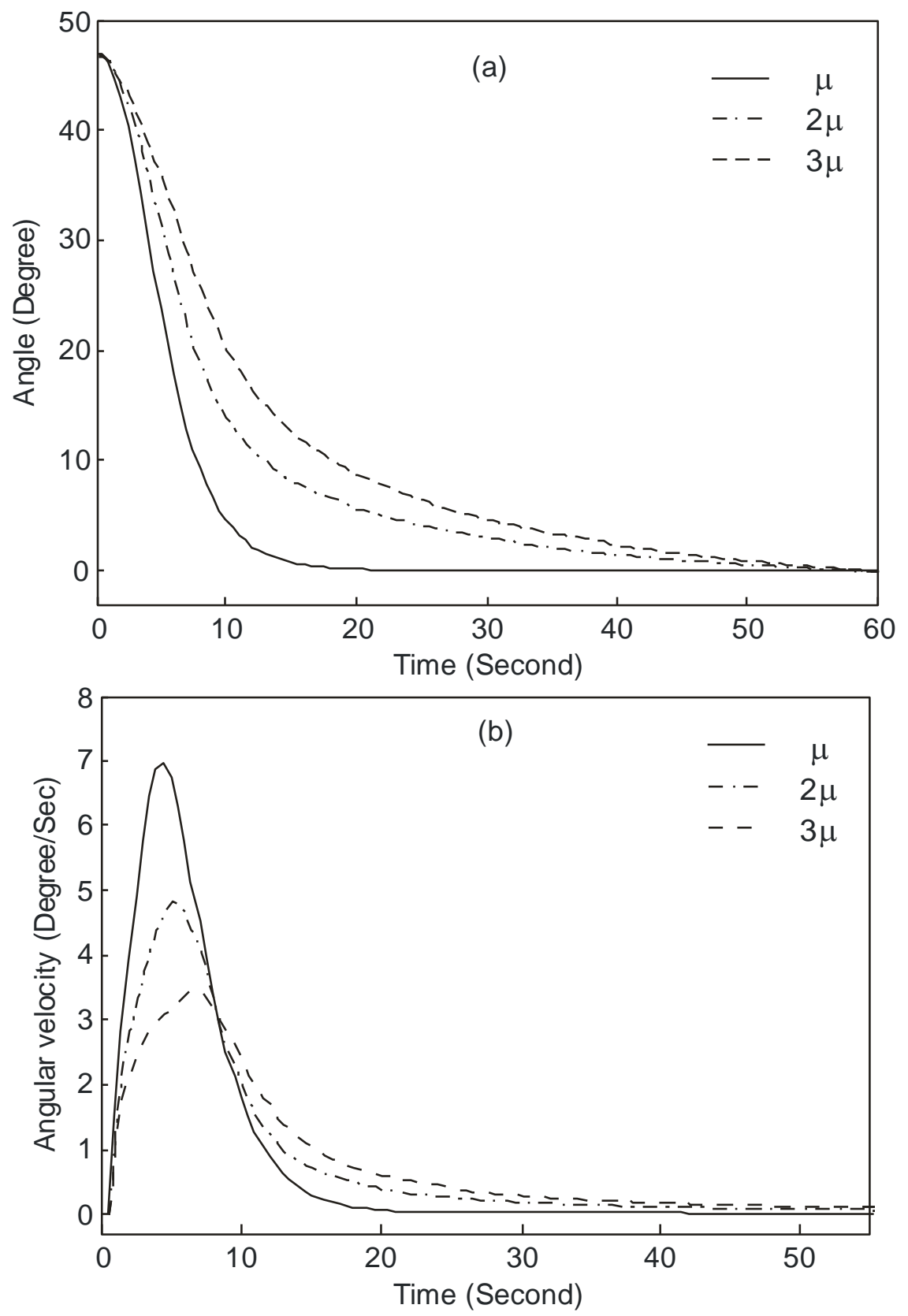

Figure 5. 

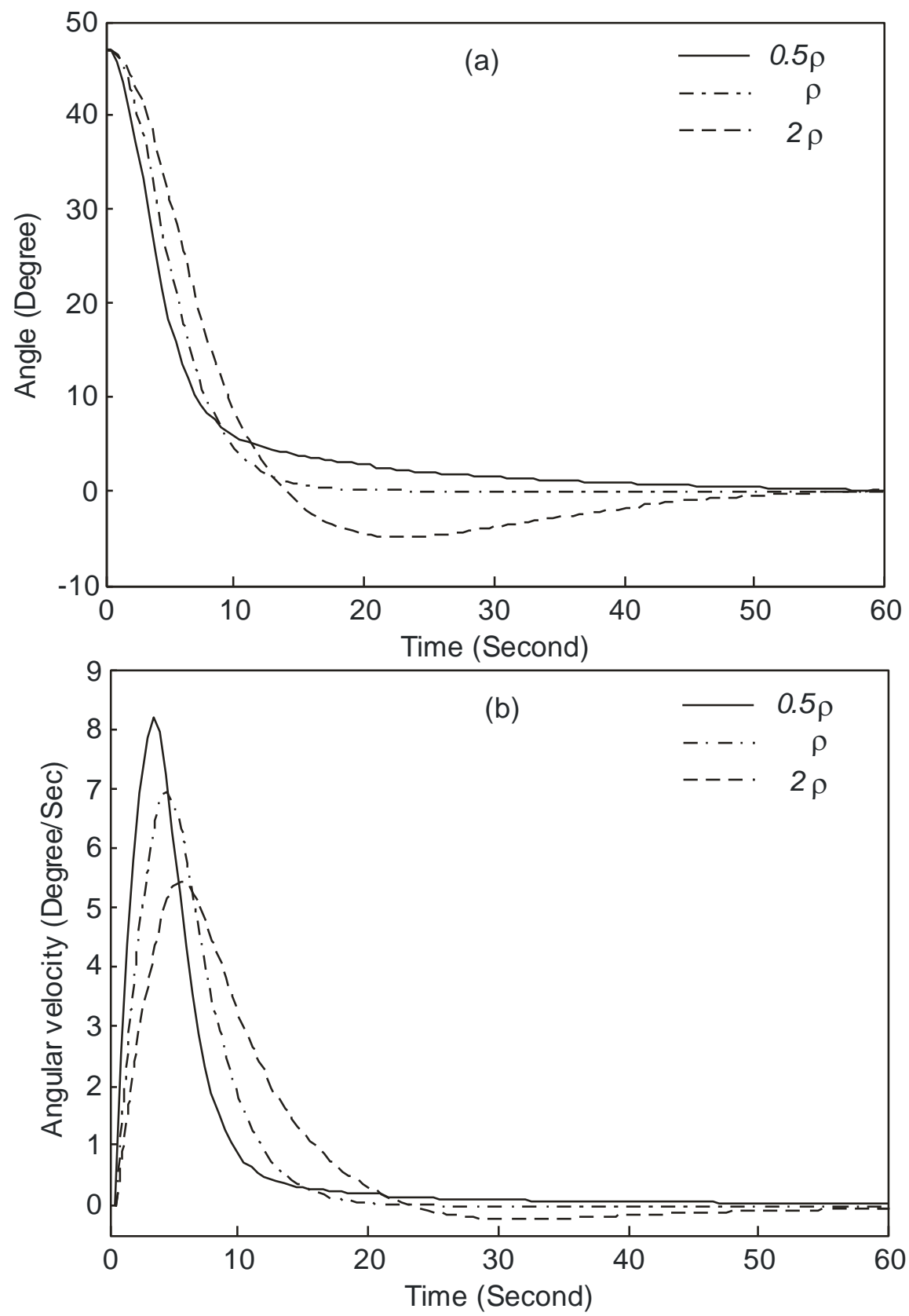

Figure 6. 

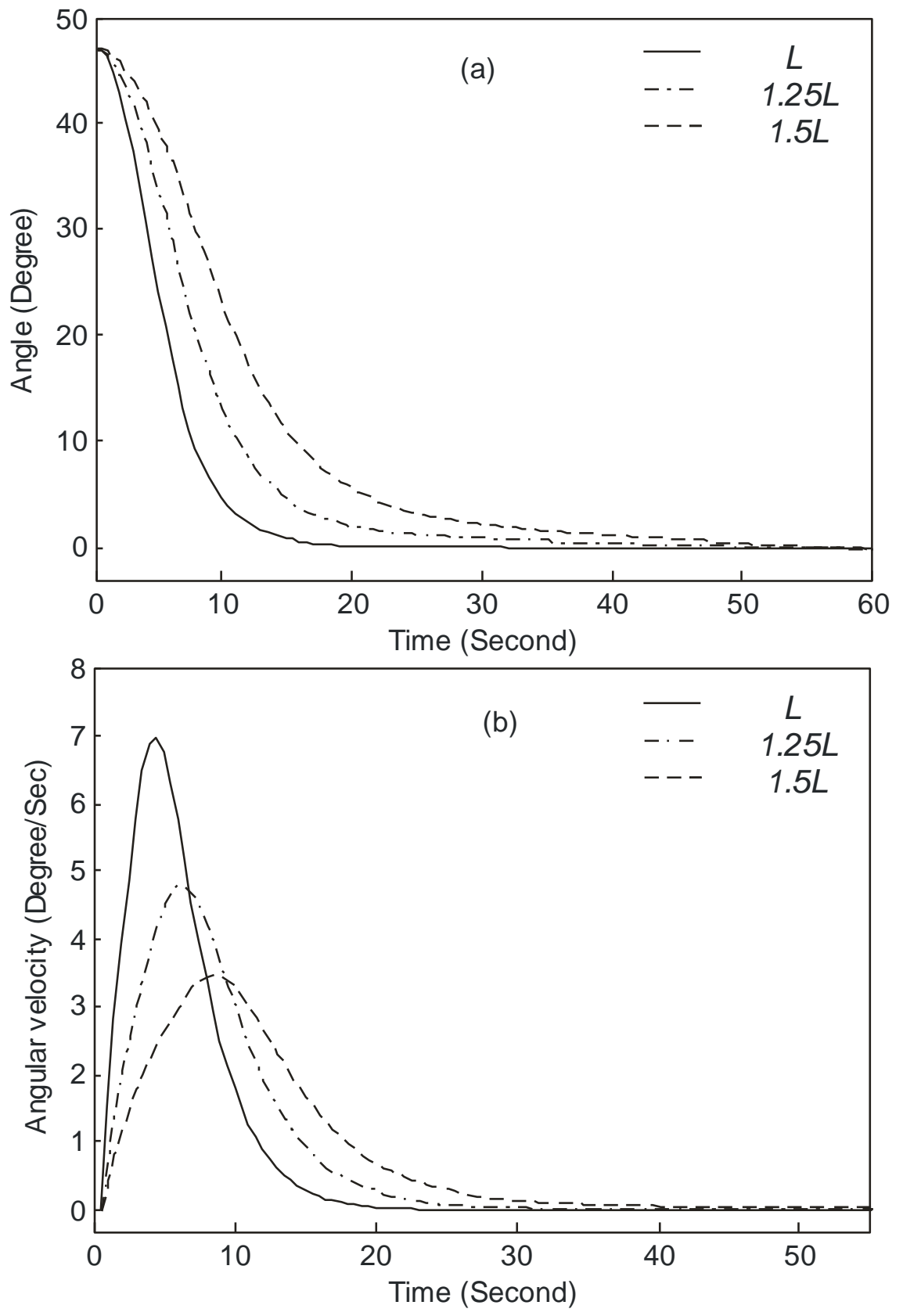

Figure 7. 

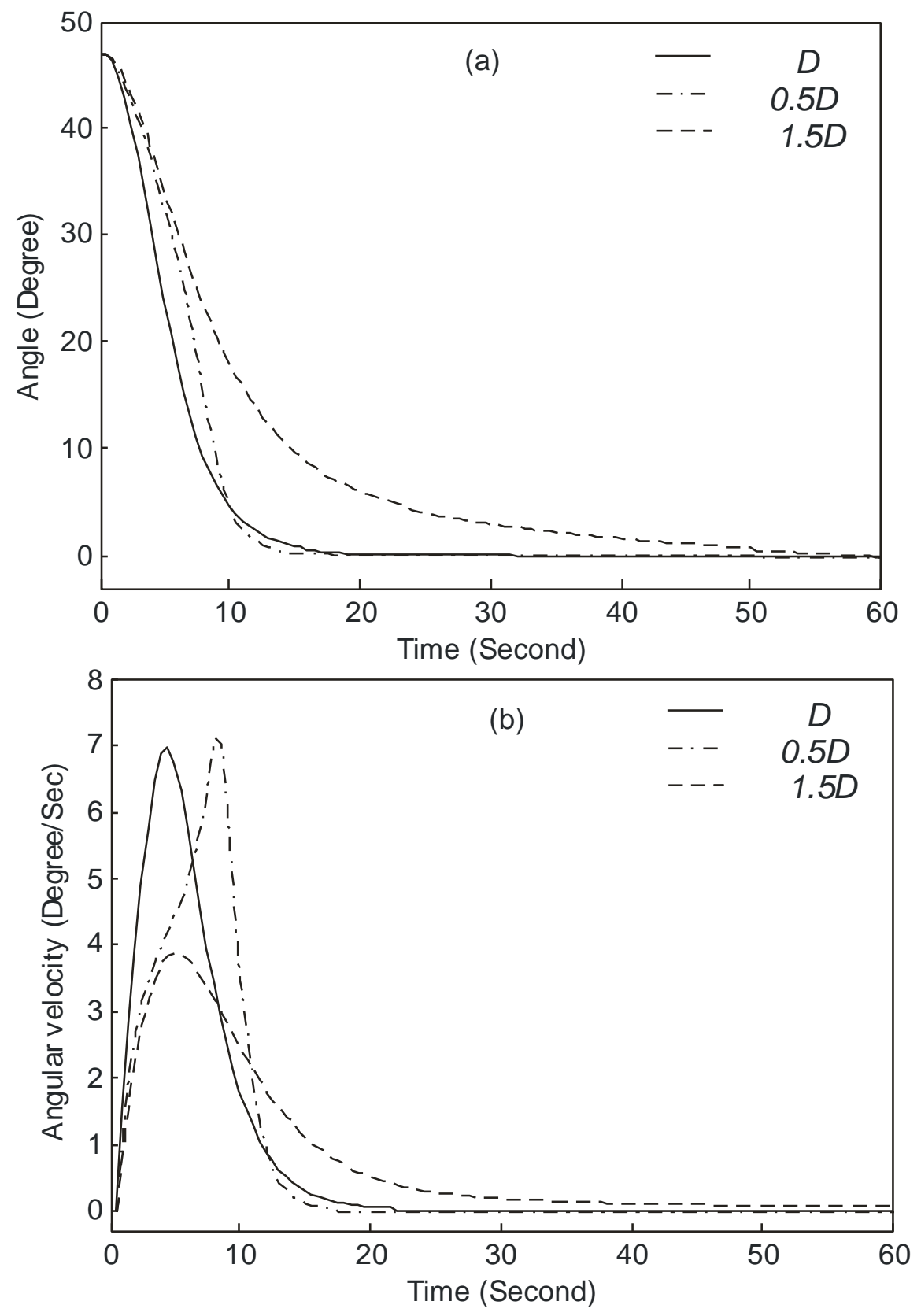

Figure 8. 


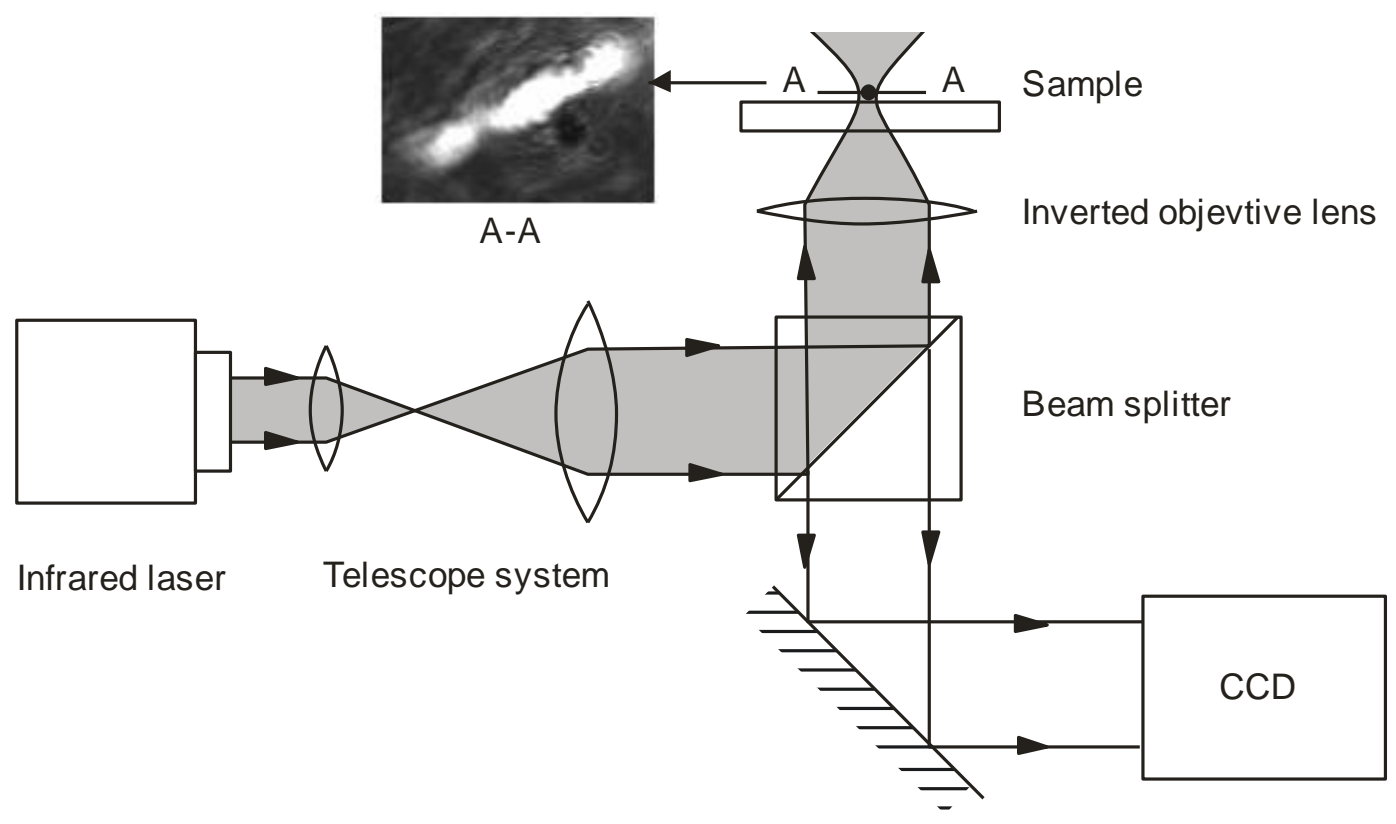

Figure 9. 

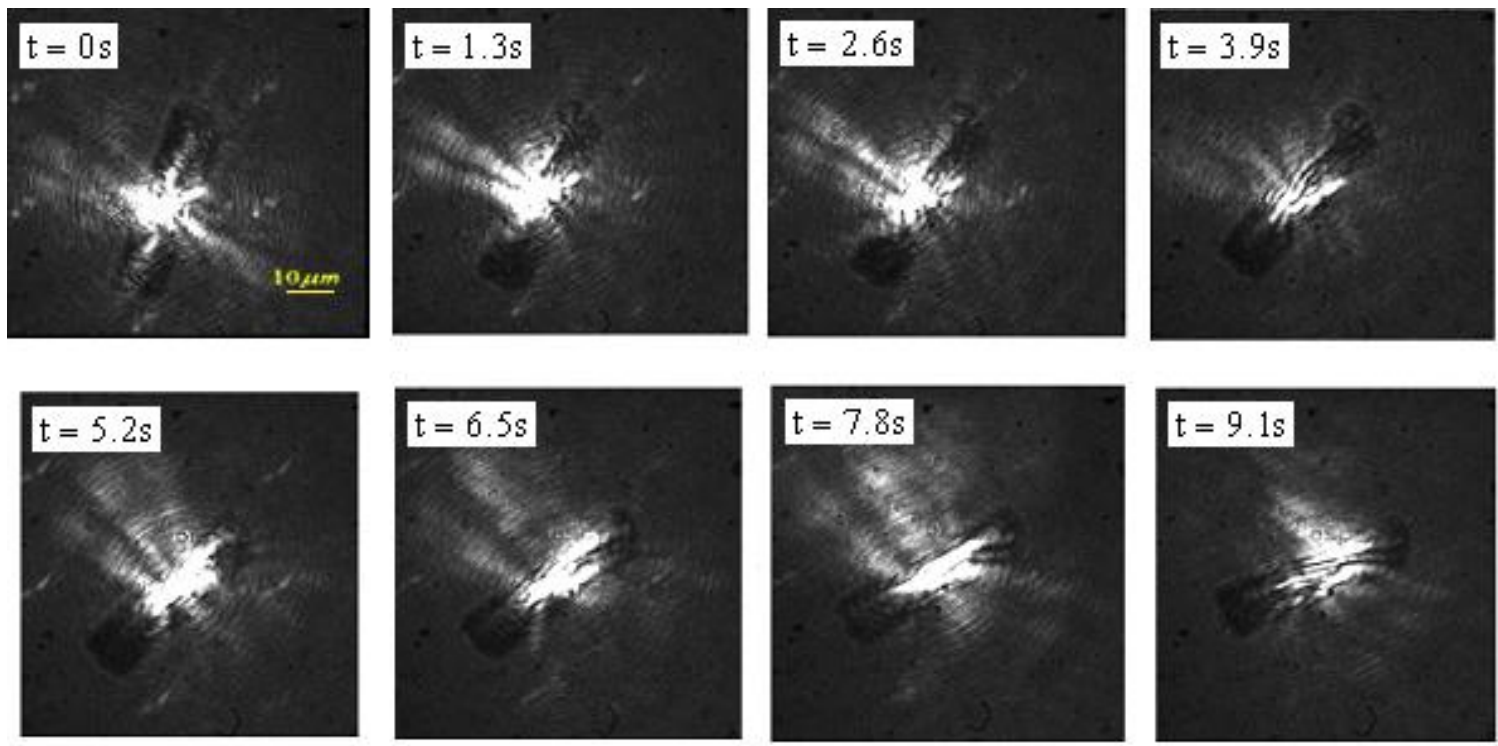

Figure 10. 

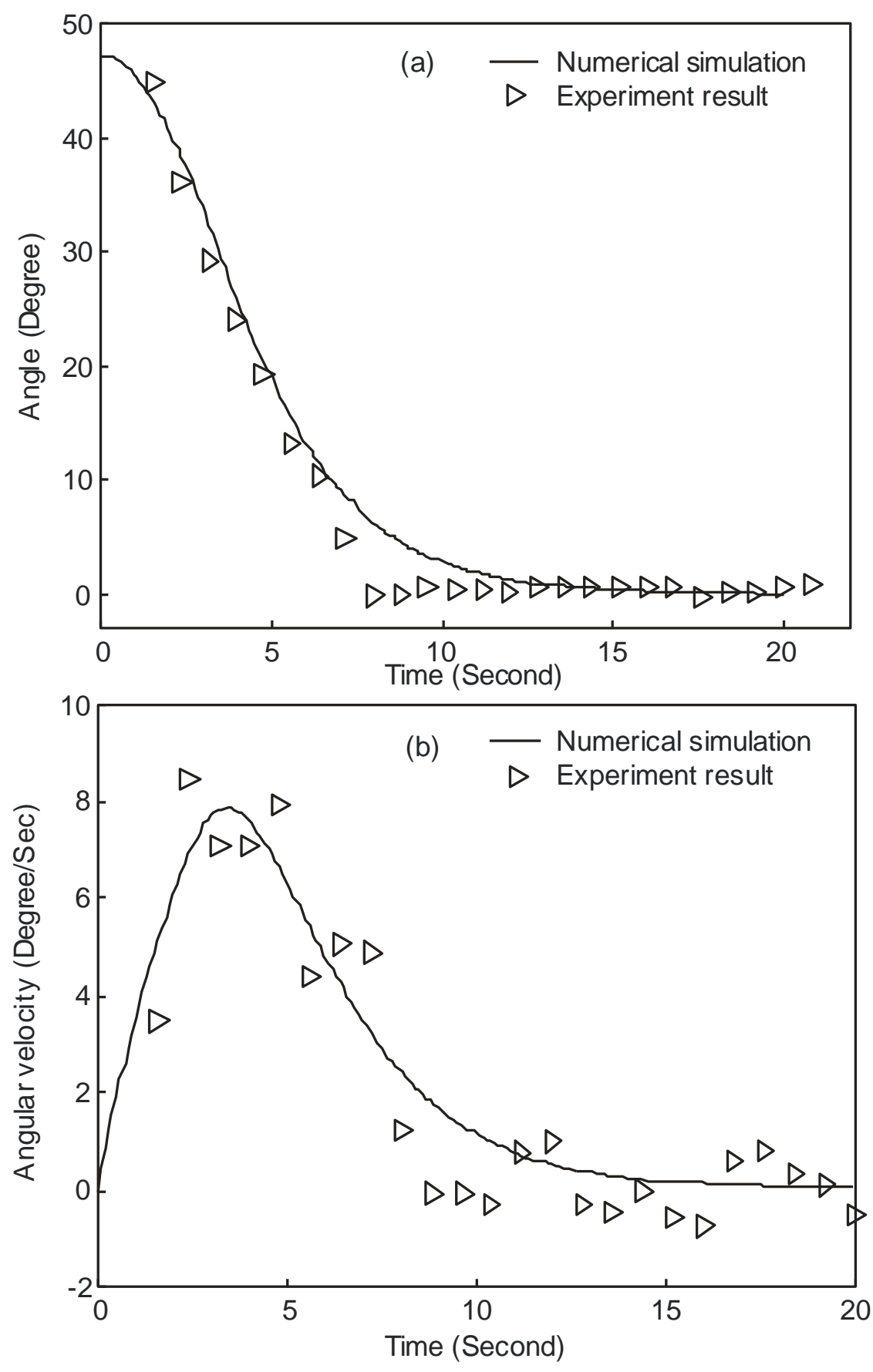

Figure 11. 